\title{
76. NEW VARIABLES IN THE LARGE MAGELLANIC CLOUD
}

\section{J. Landi Dessy}

Observatorio Astronómico, Córdoba

This work was performed in the same way as that corresponding to the Small Cloud (Landi Dessy 1959a, 1959b). Two hundred and seventy-seven new variables have been discovered, whose positions are shown by arrows in Figure 1. The pairs of plates used for blinking have from $80^{\mathrm{m}}$ to more than $1000^{\mathrm{d}}$ intervals.

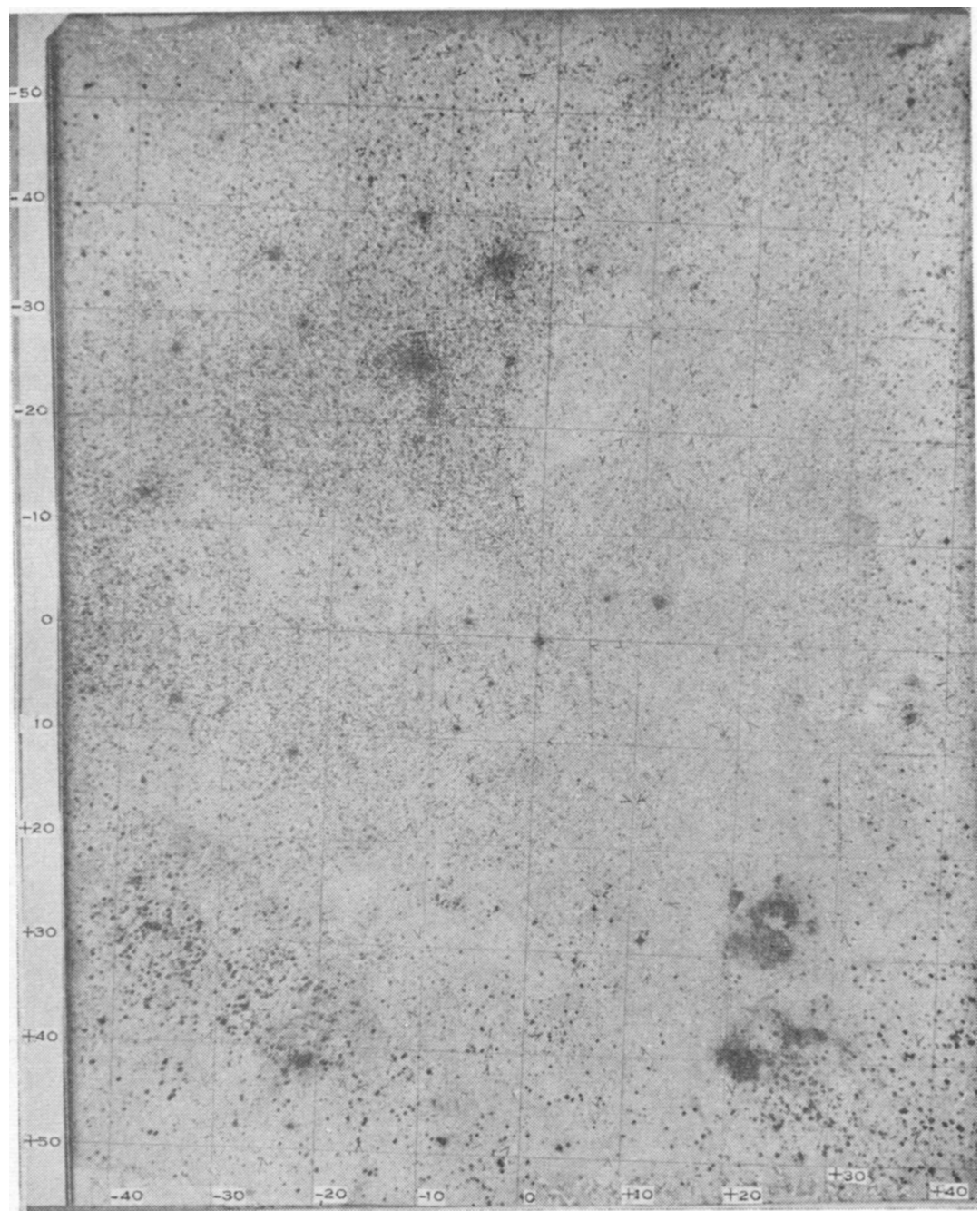

Fig. 1.-Region of the Large Cloud. 
Although the material is more abundant and better distributed than for the Small Cloud, not a single variable was found with a period smaller than $1^{d}$, neither cepheid nor eclipsing. As the observational material was distributed with the view of determining periods smaller than $1^{\mathrm{d}}$, only a small number of new periods could be determined.

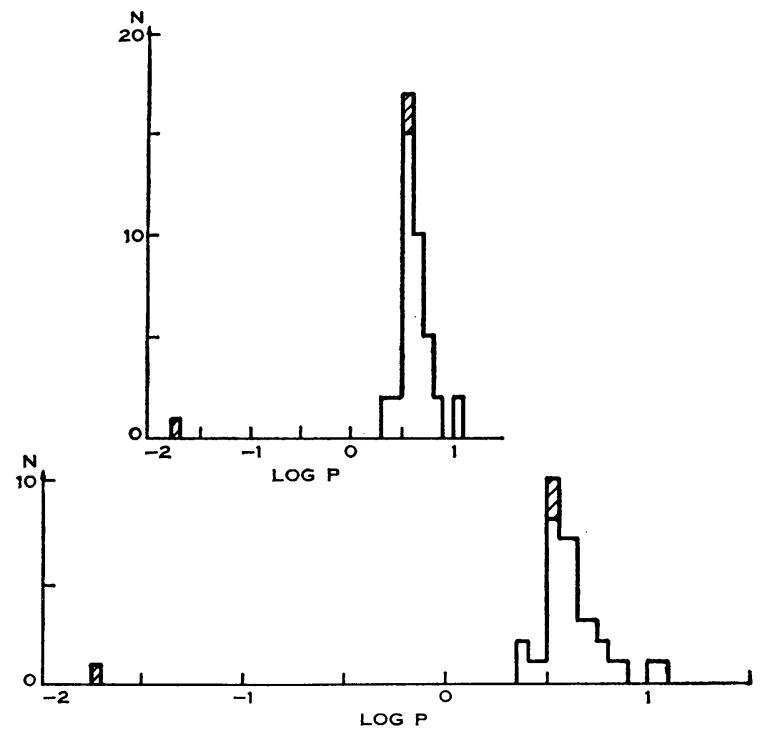

Fig. 2.-Histogram of region " $A$ " of the Large Cloud.
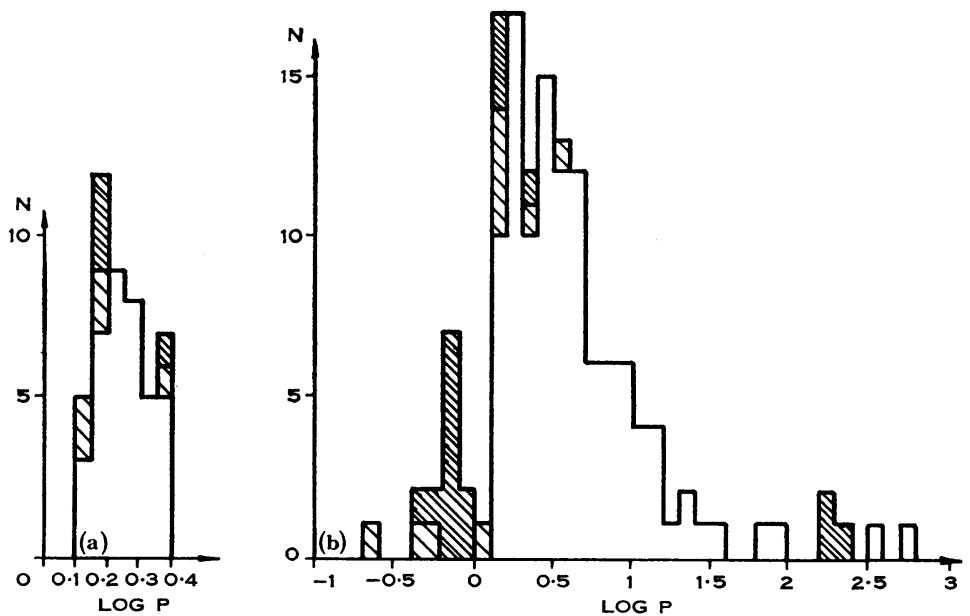

Fig. 3.-Histogram of region " $a$ " of the Small Cloud.

The histogram for the Large Cloud (Fig. 2)-number of variables versus $\log P$-shows that the distribution of the periods is different from that of the Small Cloud (Fig. 3). In the Small Cloud the most frequent period is 1.5 ; while in the Large 


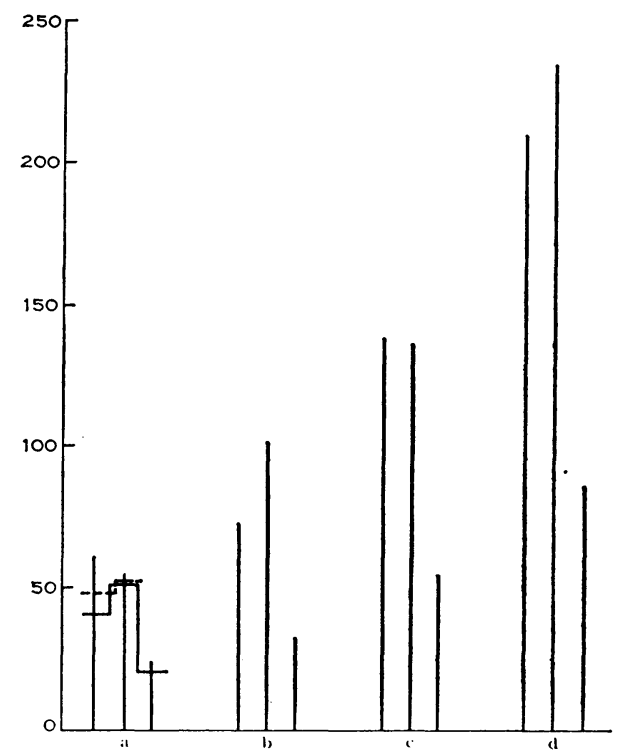

Fig. 4.-Correlations of periods and blinks in the Small Cloud. (a) Distribution of Harvard variables with known periods (some of the periods were established in Córdoba); (b) distribution of all Harvard variables with or without known periods; (c) distribution of all Córdoba variables; $(d)$ distribution of all the known variables of the region.

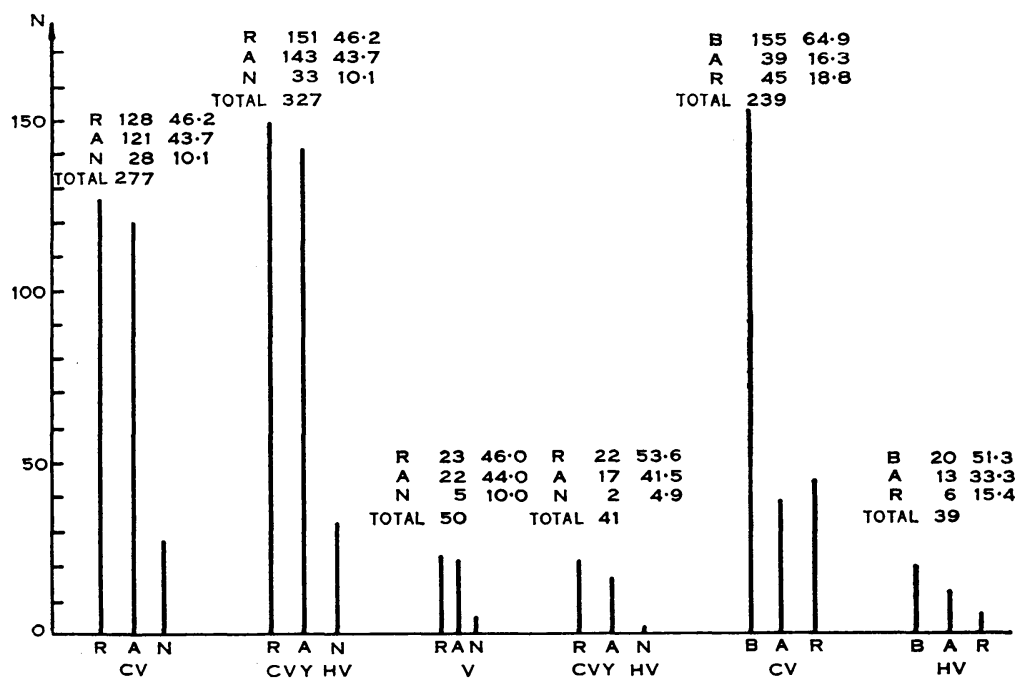

Fig. 5.-Region of the Large Cloud. First graph, distribution of all Córdoba variables; second graph, distribution of all Córdoba variables and Harvard variables; third graph, distribution of Harvard variables; fourth graph, distribution of Harvard variables and Córdoba variables with known periods; fifth graph, colour of Córdoba variables (B, blue; A, yellow; R, red); sixth graph, colour of Harvard variables. 
Cloud-at least in the region investigated-the most frequent period is $3 \mathbf{d} 6$, and no variables appear with periods less than $1^{\mathrm{d}}$. The only one which was found is clearly a galactic object.

This result is confirmed by comparing Figures 4 and 5. In Figure 4, concerning the Small Cloud, the first vertical line of each group represents the observed number of variables from pairs with intervals between $80^{\mathrm{m}}$ and $7^{\mathrm{h}}$; the second line represents stars observed in pairs with intervals between $1^{\mathrm{d}}$ and $2^{\mathrm{d}}$ and not observed in preceding pairs; the third line corresponds to stars observed only in pairs with intervals of 20 or more days. Figure 5 is similar except that it refers to stars of the Large Magellanic Cloud. The first group of lines contains only variables found at Córdoba, the second group variables found at Córdoba and at the Harvard Observatory, the third Harvard variables with known periods, and the fourth Harvard and Córdoba variables with known periods.

One sees that the new variables have altered considerably the distribution of periods for the Small Cloud, but not for the Large. In Figure 5 the fifth group of lines illustrates the colours of Córdoba and the sixth those of the Harvard variables. The proportion of white variables is $65 \%$, of the yellow ones $16 \%$, and of the red ones $19 \%$. Apparently the region is somewhat obscured, but only partially, and it does not seem to us that this can have an influence upon the distribution of the periods.

\section{References}

Landi Dessy, J. (1959a).-Ból. del I.M.A.F. Córdoba 1 : No. 2.

LANDi Dessy, J. (1959b).-P.A.S.P. $71: 435-40$.

\section{Discussion}

Heard: Can the speaker comment on the apparent scarcity of eclipsing variables which he has noted in the Large Cloud?

Landi Dessy: In the SMC we found a number of eclipsing variables around 17th magnitude. Here in the LMC we have found none.

\section{REVIEW OF MAGELLANIC CLOUD PROBLEMS}

\section{A. D. Thackeray}

\section{Radcliffe Observatory}

It must be obvious to all of us that no such Symposium on the Magellanic Clouds as we have been holding over the past few days would have been possible 10 years ago. At that time, radio work on the Clouds had scarcely begun. de Vaucouleurs, Buscombe, and Gascoigne were preparing their summary on Cloud problems as a preliminary to work with the Canberra 74-inch reflector, while the first results of work with the Radcliffe reflector were slowly beginning to emerge. Even five years ago, such a Symposium could scarcely have been fruitful, but the interest of many northern astronomers in the Clouds had certainly been excited. Now there are far more instruments and astronomers concentrated on Magellanic Cloud problems than ever before and the string of papers presented to this Symposium with lively discussions gives striking evidence of their activity. 